\title{
In-Space Manufacturing Baseline Property Development
}

\author{
Tom Stockman ${ }^{1}$, Judith Schneider ${ }^{1}$, Tracie Prater $^{2}$, Quincy Bean ${ }^{2}$, Nicki Werkheiser ${ }^{2}$ \\ ${ }^{1}$ University of Alabama in Huntsville; \\ 301 Sparkman Drive NW, Huntsville, AL 35899, USA \\ ${ }^{2}$ NASA-Marshall Space Flight Center; \\ Redstone Arsenal, Huntsville, AL 35812, USA
}

\begin{abstract}
The In-Space Manufacturing (ISM) project at NASA Marshall Space Flight Center currently operates a 3D FDM (fused deposition modeling) printer onboard the International Space Station. In order to enable utilization of this capability by designer, the project needs to establish characteristic material properties for materials produced using the process. This is difficult for additive manufacturing since standards and specifications do not yet exist for these technologies. Due to availability of crew time, there are limitations to the sample size which in turn limits the application of the traditional design allowables approaches to develop a materials property database for designers. In this study, various approaches to development of material databases were evaluated for use by designers of space systems who wish to leverage in-space manufacturing capabilities. This study focuses on alternative statistical techniques for baseline property development to support in-space manufacturing.
\end{abstract}

\section{Introduction}

The 3D Printing in Zero G technology demonstration mission, is responsible for providing fast, local solutions to hardware needs during spaceflight operations. The capability to produce parts in flight could vastly improve sustainability for long duration missions and reduce launch costs. As part of the technology demonstration mission, a fused deposition modeling (FDM) 3D printer en the ISS was installed on the International Space Station in fall 2014. The initial set of builds consisted of 20 parts, which ranged from mechanical test coupons to functional tools such as a hex head socket and ratchet.

In order for ISM to progress and develop further manufacturing capabilities, baseline material properties must be established. The development of material properties is a familiar challenge to the additive manufacturing (AM) community as a whole. In parallel with the FDM processing for ISM, NASA is also developing specifications for metal based AM processing and looking for transferable concepts, procedures, methodologies, and standards. NASA's current approach involves the development of a probability reference distribution which is capable of evolving to reflect changes/improvements in the AM process. 
Limitations on astronaut crew time impart a unique challenge by significantly limiting the number of test coupons that can be produced to quantify material properties and verify the materials produced by the process are of sufficient quality. In addition to the time limitations, developing a design allowables database for AM in general must account for the complex relationship between the build parameters and the resulting properties. Thus the goal of materials characterization for AM is to provide an engineering understanding of material behavior under a range of conditions. Statistical techniques must be utilized to effectively analyze limited data, especially with regard to properties of ISS printed components.

\section{Design Allowables Approach}

There are processes where traditional approaches to allowables development are appropriate, but these processes have characteristics that are not generally associated with AM at this point in time and include:

1) Well established material and manufacturing method, product form, and product thickness.

2) Governing of the production process/manufacturing technique by an industry or governmentquality specification.

3) Utilization of a closed loop and control feedback/in situ process which incorporate identified quality indicators.

4) Failure modes for materials are well-understood.

NASA's standard materials and processes requirements for spacecraft components combine the various standards individually developed for different material families into NASA-STD-6016 [1]. Common to all material families is the establishment of design allowables (or tolerance band).

Typically design allowables are created by systematically testing a set number of samples from various lots. These lots can be defined on the basis of material processing such as temperature, build direction, cross sectional areas, etc. The results of these tests are statistically analyzed to define a baseline property referred to as an allowable. The highest level or most stringent on the number of specimens and their interpretation is the A-basis, or A-value. The A-basis design allowables are defined on the basis of a 95\% lower confidence level bound applied to the $1^{\text {st }}$ percentile of a specified population of measurements $[2,3]$. This provides a tolerance band for the acceptable materials properties.

B-basis, or B-value design allowables are generally applied to the design of non-structural or redundant materials. B-basis allowables are defined using a 95\% lower confidence level bound applied to the $10^{\text {th }}$ percentile of a specified population of measurements $[2,3]$.

An S-basis allowable, which is simply a minimum design value specified by a governing industry or government specification, does not have an associated tolerance bound. If neither an A-basis nor B-basis database has been developed for a material, a statistically based S-basis allowable for a material can be used. Per NASA-STD-6016 [1], S-basis allowables are not typically used in primary structures or fracture-critical hardware without justification and documentation. 
Per NASA-STD-6016, NASA can create a material usage agreement (MUA), to provide technical rationale for use of the materials which do not have A-basis or B-basis allowables such as those with an S-basis allowable. In these instances, the hardware developer must provide a plan describing the material property development philosophy and provide detailed insight into how the material design properties will be determined. This plan must also include information on statistical approaches.

Allowables cataloged in or derived using the procedures in NASA-STD-6016 are widely accepted among the aerospace design community. The number of test specimens required for Abasis and B-basis allowables varies among the various families of materials. Metallic materials require a minimum sample size of 100 obtained from 10 lots with 10 samples each [3]. If the distribution is non-parametric (i.e. does not fit a normal distribution), at least 300 samples are required. In contrast, polymeric composites only require 30 samples for an A-basis allowable. Thus materials made using FDM have the additional complication of not having standards to guide the generation of an A-basis or B-basis design allowables database.

Components manufactured using AM processes, either for metals or polymers, are highly process dependent. Composites are in many ways similar to AM produced materials. In both cases the properties are highly process dependent, often anisotropic, and very sensitive to test specimen geometry and test technique. Thus MIL-HDBK-17 details standardized and validated methods for establishing composite allowables that are slightly different from those established by MIL-HDBK for metals in terms of number of samples, number of lots, and other constraints and rules. MIL-HDBK-17 also specifies design allowables for the constituents of the polymeric composite which may have applicability to the FDM polymeric materials.

Another limitation of NASA-STD-6016 type methodologies for AM is that once material property development activities are complete, little opportunity exists to revisit the established allowables database. The risk inherent in using this approach for an evolving process, such as $\mathrm{AM}$, is that once the established design allowables no longer reflect the process or the materials produced, the development work needs to be repeated. Without methods to update the allowables as the AM process matures, designers will continue to use values that do not represent the materials being produced.

AM broadly challenges the established allowables development philosophy. Traditional allowables approaches are not suitable for in-space manufacturing and specifically development of material design values for materials produced using the 3DP FDM hardware currently on the ISS. The ISM team is limited in the scope of a fully executed allowables development program based on the guidelines of the design handbooks [2,3]. Considering the variability and evolving nature of the AM process, the data derived from such an effort may not retain meaningfulness in the long-term.

At this time, the parts selected for fabrication using the 3DP FDM process on the ISS have low consequences of failure. To help develop the procedures and methodology, a complementary 
effort is focused on structural modeling at the macroscale. Given these constraints and considerations, an alternative approach using statistical techniques for baseline property development to support in-space manufacturing may provide a better solution for confidence in establishing a design allowables database.

\section{Modified Approach}

A two phase study is proposed to statistically establish material properties for 3DP using FDM. Phase I of this work seeks to understand the sensitivity of parts produced using the process to manufacturing process variables. This can be accomplished by carefully crafting a set of screening experiments that will assess whether and to what degree, the first-tier material properties are a function of feedstock material, build orientation, filament layup, test temperature, and printer. The study seeks to broadly answer the following questions:

1) How do build orientation and layup impact material properties?

2) What degradation or improvement in properties can be expected based on the test temperature, and, by extrapolation, the use temperature of the component?

3) What amount of variability in material properties can be expected when two parts with the same feedstock, build orientation, and layup are printed on different printers?

4) Does feedstock manufacturer impact material properties of the as-built part in an engineering significant way?

The Phase 1 study is an exploratory test that includes: 35 sets of tensile, compression, and shear specimens. 3 temperatures, 5 combinations of build orientation and filament layup, and 3 feedstock materials, printed on 2 printers. The primary goal of the first phase is to closely examine specimens printed in space and compare them both quantitatively and qualitatively to the same specimens printed on the ground. Each of the ISM specimens has a twin built on a twin machine on the ground. These specimens will go through mechanical testing (tensile, compression, etc) along with measurements for mass, volume, and tolerancing. The specimens will also be subject to x-ray radiography to give a visual inspection of the internal structure. If the specimens printed in space are found to have very little difference from specimens printed on the ground, then further material characterization can be performed on the ground where crew time and transportation to and from ISS are not needed. The sensitivity analysis/screening experiment will indicate which factors have a statistically significant impact on material quality and the ISM team will use this data to develop a Phase II Design of Experiments (DOE) protocol/experimental matrix.

Data from the Phase II DOE will be modeled using regression techniques. The response surface generated by the regression models will be used to define a statistical tolerance region which bounds characteristic material properties for a given combination of temperature, build orientation/layup, printer, and feedstock. While nontraditional, this approach represents the best 
fit for the current needs thereby allowing the execution of a material property development program that will:

1) Enable the development of an efficient test plan that will minimize the number of test articles yet still provide validated information about material behavior that can be used for design and analysis.

2) Generate analyzable data.

3) Calculate basis values for a combination of material and processing characteristics that will reduce reliance on engineering judgment.

The approach will leverage DOE techniques to optimize the value of information and allow material property development and materials characterization activities to occur simultaneously. Subsequent investigations such as assessing machine variability, lot to lot variability, the effect of feedstock, etc., can be performed as needed through additional/follow-on DOEs. Because DOE enables variation of several factors at a time, the technique is more cost-effective and less experimentally intensive than the "one piece at a time" approach.

\section{Summary}

ISM is a significant advancement to in space capabilities. However, certification and development of material properties is made difficult by the newness of the technology, the high process dependency of the technology, and a limited capability to produce large numbers of samples. Using a phased approach a database baseline can be established to develop usable properties. These properties will not necessarily be representative of traditional A-basis/B-basis allowables but will still be sufficient for the design and production of 3D printed parts in space.

\section{Acknowledgments}

\section{References}

[1] "Standard materials and processes requirements for spacecraft," NASA-STD-6016, July $11,2008$.

[2] Composite Materials Handbook: Vol. 1 Polymer matrix composites guidelines for characterization of structural materials, Pub. Department of Defense, $M I L-H D B K-17-1 F$, June 17, 2002. Now published as the Composites Materials Handbook (CMH)-17.

[3] Metallic materials and elements for aerospace vehicle structures, Pub. Department of Defense, MIL-HDBK-5J, January 31, 2003. Now published as the "Materials Properties Development and Standardization (MMPDS)." 